\title{
Planar Center-Fed Leaky-Wave Antenna Arrays for Millimeter Wave Systems
}

\author{
Yu.B. Nechaev and D.N. Borisov \\ Voronezh State University, Voronezh, Russian Federation
}

\author{
A.I. Klimov \\ Voronezh Institute of the Interior of Russia, Voronezh, Russian Federation \\ I.V. Peshkov \\ Bunin Yelets State University, Yelets, Russian Federation
}

\begin{abstract}
Two kinds of planar center-fed leaky-wave antenna arrays for millimeter wave systems are presented. The antenna arrays consist of a printed one-dimensional periodic metal strip grating on a grounded dielectric waveguide and designed for broadside radiation. The first antenna array has a hybrid structure with a feeding device for the planar dielectric waveguide, represented by a center-fed linear slotted waveguide array. The second antenna array has a simple single-layer structure with a feeding device, formed by a comb line using the central strip of the grating. The investigated examples of both kinds of arrays exhibit low return loss at frequencies of broadside radiation. The radiation efficiency of antennas exceeds $50 \%$ at frequencies up to $37-37.5 \mathrm{GHz}$, while the antenna gain is above $30 \mathrm{dBi}$.
\end{abstract}

KEYWORD: planar antenna array; surface waves; leaky waves; metal strip grating; grounded dielectric waveguide

\section{INTRODUCTION}

Significant progress in development and practical application of compact electronic components and devices for centimeter and millimeter-wave radio communication systems, wireless computer networks, radars and sensors necessitates the development of new compact high gain and low cost antennas. However, design of planar high gain and high efficiency millimeter wave antennas still remains a serious challenge in antenna engineering [1-2]. Many planar antenna arrays using conventional transmission lines suffer from the increasing dielectric and conductor loss at millimeter wavelengths [2]. The leading candidates for highgain planar antennas are various kinds of waveguide slotted antenna arrays with the antenna gain more than $30 \mathrm{dBi}$ at $60 \mathrm{GHz}$ and higher frequencies while their radiation efficiency is not less that $60 \%$ [2]. At the same time, leaky-wave antenna arrays (LWAA) [3-7] are also promising candidates for millimeter wave applications, because of such advantages, as a simple structure and a quite high radiation efficiency, as well as a low-cost printing technology of fabrication. The size of LWAA and its radiation properties directly depend on the design and performance of the feeding device for dielectric waveguide (DWG), which is a base part of many leaky-wave structures. In developed so far dielectric
LWAA DWG has been excited by a rectangular waveguide, a sectoral horn, a parabolic reflector, a waveguide slot, and a linear array of radiating elements similar to slots or microstrip stubs [4-7]. Despite considerable progress in designing compact and efficient LWAA, obviously there is still room for improvement of the broadside LWAA, concerning decrease of return loss and side lobe level (SLL) of the radiation pattern, increase of directivity, radiation efficiency and gain, minimization of the antenna size and weight.

This report presents two more technical solutions of broadside LWAA, composed of DWG and planar diffraction grating [8-9]. The proposed antennas have center-fed single dielectric layer structures, designed to provide low return loss and effective linearly polarized broadside radiation.

\section{PRINCIPLE OF OPERATION}

The principle of operation of the LWAA, considered in this report, is based on a well-known effect of surface electromagnetic waves transformation, while propagating along the DWG, into leaky waves, radiating from the structure due to scattering of the surface waves on a periodic diffraction grating [3], [7]. The proposed LWAA are supposed to operate on the $-1^{\text {st }}$ spatial harmonic of the diffraction field and 
all their parameters are chosen and calculated in a way to perform as resonant diffraction structures in vicinity of Bragg diffraction of the second order and to form a narrow broadside beam. For such a purpose, parameters of the radiation structure are to satisfy a known condition $c / v\left(\lambda_{0}\right)=\lambda_{0} / d ; c-$ velocity of light in the free space, $v\left(\lambda_{0}\right)$ - phase velocity of surface waves, $\lambda_{0}-$ wavelength of radiation, $d$ - period of grating [3], [7].

The first of two proposed center-fed LWAA [8] (Figure 1), developed for 36-37.5 GHz frequency range, consists of a grounded DWG 1, a periodic diffraction grating (forming the radiation aperture with the size of $L_{x} \times L_{y}$ ) composed of two arrays of parallel metal strips 2 (having common central strip), and a feeding device 3 for the DWG. One of the embodiments of the feeding device can be described as a rectangular waveguide with coupling slots, cut in its upper broad wall [8]. In the particular design, the feeding device is formed by a metal rectangular trough waveguide with an input rectangular slot in the center of the bottom, and a linear array of $L$ shaped slots, cut in the metal ground plate of DWG underneath the central strip of the grating, as shown in Figure 1.

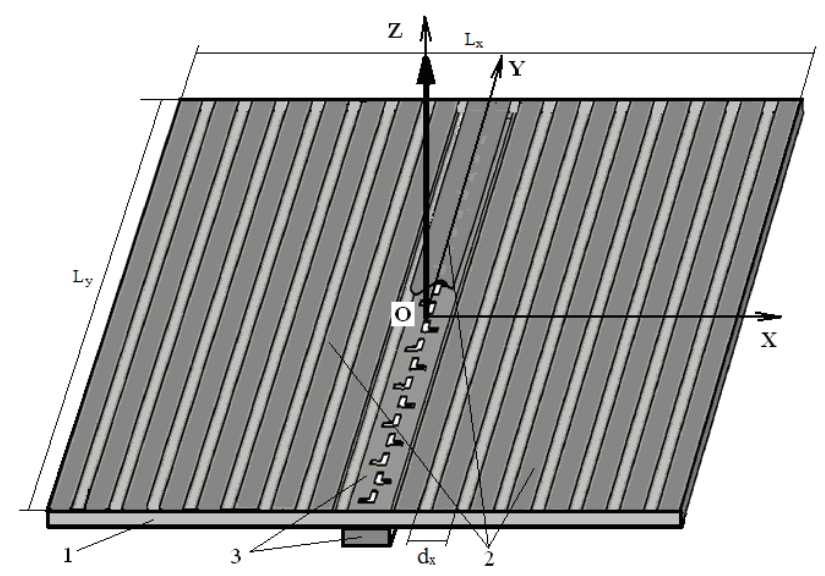

Figure 1. A general view of the first antenna design

When an excitation is applied to the input slot, TE10 electromagnetic waves propagate along the trough in opposite $( \pm \mathrm{OY})$ directions. Due to the presence of L-shaped slots, periodically situated at the distance of a half of TE10 mode wavelength in the trough at the central design frequency, leakage of power into DWG takes place, causing intensive excitation of TM surface waves, propagating in opposite $( \pm \mathrm{OX})$ directions along the DWG. Diffraction of these waves on the periodic arrays of metal strips results in radiation into surrounding space, and, since leaky waves from both left and right parts of the structure are in phase towards $+\mathrm{OZ}$ direction, intensive broadside radiation arises, producing a single main beam in the radiation pattern. To eliminate a problem of sharp decrease of radiation efficiency at broadside, inherent to many open periodic structures operating in the vicinity of the open stop-band [6], [10], in [8] has been suggested to choose the width of the central strip of the grating to be close (or equal) to the period $\mathrm{dx}$, both left and right parts (strip arrays) of the grating to move to the left and to the right on one quarter of $\mathrm{dx}$ and to choose the thickness of the DWG to be close (or equal) to one quarter of a wavelength in dielectric.

The first center-fed LWAA has been designed for $36-37.5 \mathrm{GHz}$ frequency range in order to obtain the antenna gain not less than $30 \mathrm{dBi}$. Also, various means to reduce the side lobe level of the E-plane (XOZ) radiation pattern have been tried: for instance, use of non-equidistant grating with strips of non-equal width, or use of equidistant grating with strips of constant width, but with reflecting metal walls, short-circuiting outer edges of the strips to the ground plate at the left and right borders of the DWG.

To minimize the total size of the LWAA, another design of the antenna has been proposed recently [9]. The second center-fed LWAA (Figure 2) like the first one consists of a grounded DWG 1, a periodic diffraction grating (forming the radiation aperture) composed of parallel metal strips 2 , but contains a different feeding device 3 for the DWG [9].

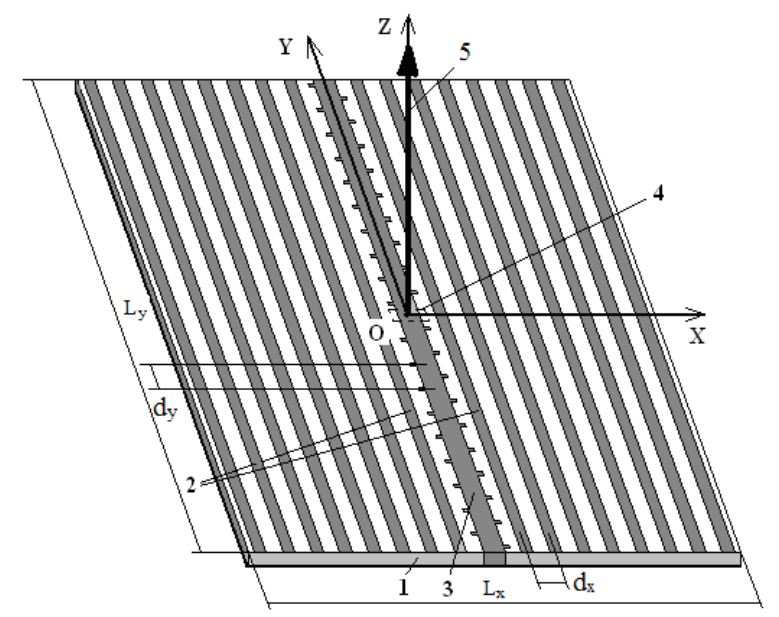

Figure 2. A general view of the second antenna design

To feed the antenna, a rectangular input slot 4 is cut in the center of the metal ground plate. The feeding device 3 is coplanar to the metal strip grating 2 and composed of the central strip of the grating with two lateral rows of rectangular metal stubs, attached to both edges of the strip, similarly to a printed comb line structure with alternate radiating stubs.

The strips and the stubs are periodically positioned along $\mathrm{OX}$ and $\mathrm{OY}$ axis with $\mathrm{dx}$ and $d_{y}$ 
spacing; the size of the radiating aperture is $\mathrm{L}_{x} \times \mathrm{L}_{y}$. The main radiation beam direction 5 is shown as a black arrow.

Corresponding to the described above principle of operation, at the design frequency of the antenna, period $d_{x}$ is to be taken equal to one wavelength of the surface waves, propagating along DWG in $\pm \mathrm{OX}$ directions; period $d_{y}$ - equal to one wavelength of waves, excited via input slot in the transmission line (formed by the central strip with lateral stubs) and propagating in $\pm \mathrm{OY}$ directions. Therefore, the main beam is normal to the radiating aperture. More precisely, the transmission line, formed by the central strip with stubs and short-circuited to the ground at both ends, performs as an input power divider. According to the above-mentioned spacing $d_{y}$, at the design frequency one part of the input power directly radiates from the structure along $\mathrm{OZ}$ axis, but the other, larger part of the input power, due to the presence of discontinuities (stubs), converts into the power of $T M$ surface waves, propagating along DWG in $\pm \mathrm{OX}$ directions. As the result of their diffraction on the strip grating, the antenna radiates in $\mathrm{OZ}$ direction with the electric field linearly polarized in XOZ plane and parallel to OX axis. Since the second proposed LWAA also utilizes a combined corporate-series feed, its operating frequency range (as for the first LWAA) is limited by the frequency-dependent beam split. However, as it will be shown further, in case of narrow-band applications (2-3) \% such LWAA can provide a rather high directivity, up to $28-30 \mathrm{dBi}$. To avoid considerable reflection and return loss at the resonant frequency, in the second proposed antenna the DWG thickness is also chosen about one quarter wave length in the media with the same relative dielectric permittivity $\varepsilon_{r}$ (for DWG supporting TM surface waves) and the width of the central strip of the diffraction grating is taken close to $d_{x}$. In such a case a quite low reflection at the input point of the antenna can be obtained - less than $-15 \mathrm{~dB}$ [8], [9].

\section{RESULTS AND DISCUSSION}

The initial parameters of the radiating aperture of the first LWAA (Figure 1, a), such as DWG thickness, strip width and spacing (period $\mathrm{dx}$ ) have been calculated using a simple mathematical model, developed for an infinite periodic structure consisting of a grounded dielectric slab with a 1Dperiodic metal strip grating. This structure has been a subject of such well-known theoretical works, as [6], [11] - [14] and analyzed in detail in reference to leaky-wave antennas and various reflective surfaces.
A commonly used approach to the analysis is based on formulation of the dispersion equation and calculation of $\beta=\beta_{0}-j \alpha-$ the complex propagation constant of the fundamental spatial harmonic. The result is further used to calculate the propagation constant $\beta_{-1}=\beta-2 \pi / d$ of the -1 st spatial harmonic and then other characteristics of the electromagnetic fields in the structure and in the surrounding space, including the main beam radiation angle [3], [6].

In this work a diffraction model is applied [11], [12], which consider scattering of a plane H-polarized incident wave by an infinite extent structure with periodic array of conductive strips of infinitesimal thickness $\Delta$, Figure 3.

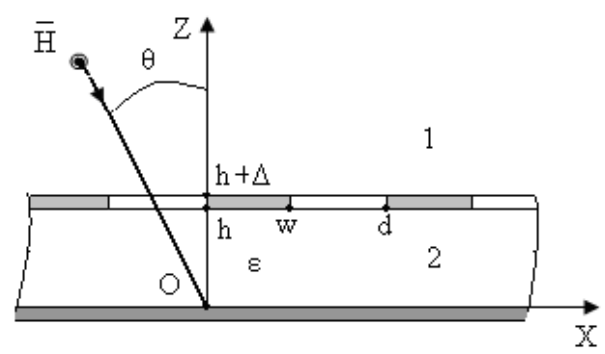

Figure 3. A single layer 1D-periodic structure

Using the point-matching technique, described in [11] and [12] for the structure shown in Figure 3, the following system of inhomogeneous algebraic equations can be obtained to calculate then the unknown complex amplitudes An of components of the scattered waves in the region 1 :

$$
\sum_{n} A_{n} \gamma_{n} e^{j \xi_{n} x}=H_{0} \gamma_{0} e^{j \xi_{0} x}, \quad 0 \leq x \leq w \text {; }
$$

$\sum_{n} A_{n}\left(1+j \varepsilon \frac{\gamma_{\mathrm{n}}}{\eta_{n}} \operatorname{ctg} \eta_{n} t\right) \mathrm{e}^{j \xi_{n} x}=-H_{0}\left(1-j \varepsilon \frac{\gamma_{0}}{\eta_{0}} \operatorname{ctg} \eta_{0} t\right) \mathrm{e}^{j \xi_{0} x}$,

$w \leq x \leq d ;$

where $\gamma_{0}=k_{0} \cos \theta$ and $\xi_{0}=k_{0} \sin \theta$ - transverse and longitudinal propagation constants of the incident wave; $k_{0}=2 \pi / \lambda_{0}$ - free-space wave number; $\theta$ - the incidence angle; $\xi_{n}=\xi_{0}+2 \pi n / d$, $n=0, \pm 1, \pm 2, \ldots$ - longitudinal propagation constant of the $n^{\text {th }}$ spatial harmonic; $\gamma_{n}=\left(k_{0}^{2}-\xi_{n}^{2}\right)^{1 / 2}$ and $\eta_{n}=\left(k_{0}^{2} \varepsilon-\xi_{n}^{2}\right)^{1 / 2}$ - transverse propagation constants of the $n^{\text {th }}$ harmonic in regions 1 and 2 respectively. At the next step the dependence of $\left|A_{-1}(\theta)\right|$ can be found, as well as the angle $\theta_{-1 m}$ providing maximum of $\left|A_{-1}\right|$, and the approximate value of the attenuation constant $\alpha_{-1}$ from , 
$\Delta \theta_{m}=\sin ^{-1}\left(\sin \theta_{-1 m}+\frac{\alpha_{-1}}{k_{0}}\right)-\sin ^{-1}\left(\sin \theta_{-1 m}-\frac{\alpha_{-1}}{k_{0}}\right)$

where $\Delta \theta_{m}$ is the half-power beam width of $\left|A_{-1}(\theta)\right|$ [15].

Use of such approach allows fast obtaining pretty accurate data on the frequency- and angle-dependent behavior of the infinite structure as a receiving leaky-wave antenna array. Numerous calculations and experiments have proven that this simple technique yields a quite satisfactory result if the incidence angle is not too close to zero (in most cases not less than $1-2$ deg.) and the number of retained space harmonics $(2 N+1) \geq 45 \ldots 50$. Therefore, the diffraction model may be successfully used for initial estimation of the necessary DWG thickness h, strip width $\mathrm{w}$ and spacing (period $d_{x}$ ).

Figures 4-5 illustrate characteristics of the first center-fed LWAA (DWG — Rogers RT/Duroid 5880, thickness $h=1.524 \mathrm{~mm}$, the aperture size $\left.L_{x} \times L_{y}=104.5 \mathrm{~mm} \times 108 \mathrm{~mm}\right)$ in $36-37.5 \mathrm{GHz}$ frequency range obtained by computer simulation using CST Microwave Studio: the reflection coefficient $s_{11}$, the antenna gain $G$ and the sidelobe level SLL of the E-plane radiation pattern (Figure 6).

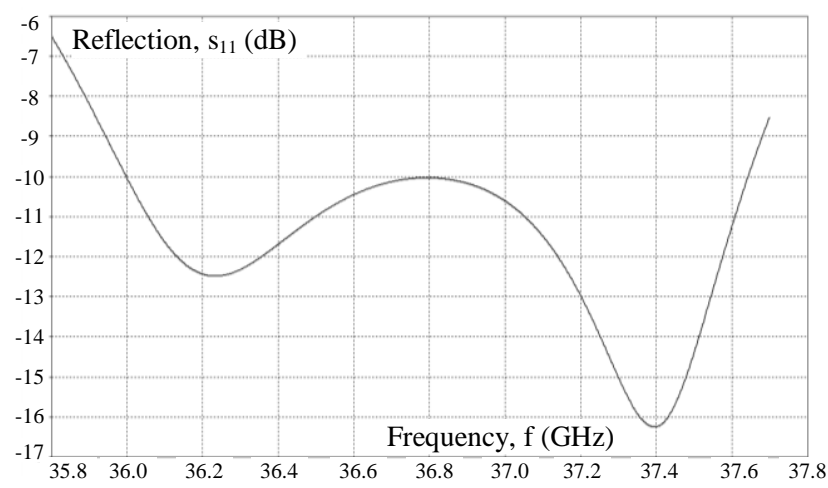

Figure 4. Simulated reflection coefficient at the input point

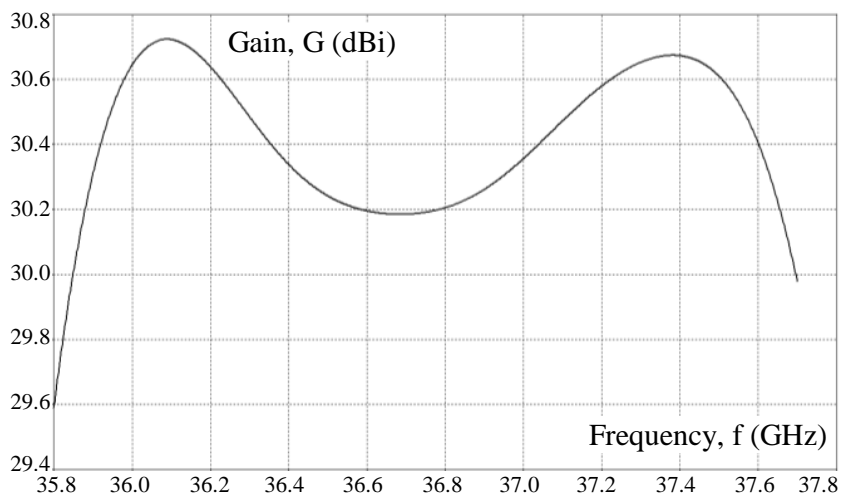

Figure 5. Simulated antenna gain

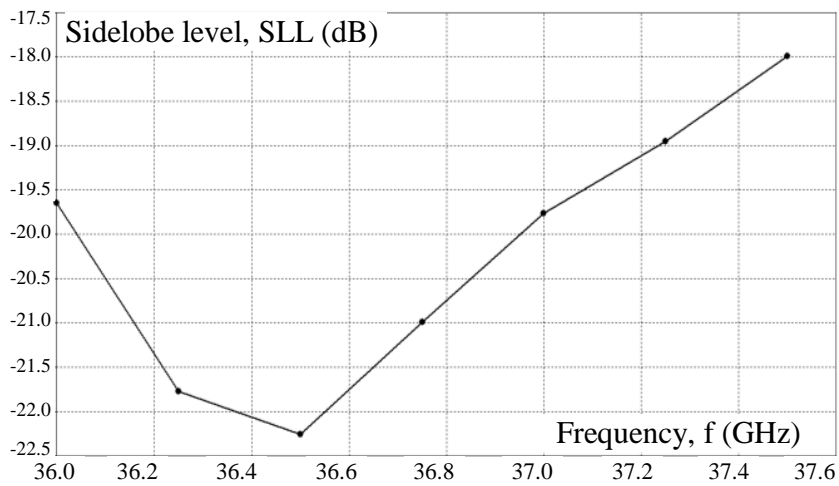

Figure 6. Simulated sidelobe level of E-plane (XOZ) radiation pattern

The antenna demonstrates a low reflection at the input point and a rather high gain while the radiation efficiency is within $49 \% \ldots .58 \%$ and the SLL in the E-plane is below $-18 \mathrm{~dB}$. The average half-power beam width in E- and H-plane is 5.5 deg. $\times 4.5$ deg. The measured and simulated characteristics of the antenna are close enough. Worth to notice that the proposed design of the antenna allows an independent control of amplitude distribution in radiating aperture, as in $E$-, as in $H$-plane, and to attain some better radiation efficiency (at least up to about $70 \%$, as reported in [8]).

Two examples of the second proposed LWAA have been designed for $24-24.4 \mathrm{GHz}$ and $36.5-$ $37.3 \mathrm{GHz}$ frequency ranges, in intention to obtain the antenna gain not less than $30 \mathrm{dBi}$. The first example with the aperture size $156 \mathrm{~mm} \times 156 \mathrm{~mm}$ (DWG - Teflon, thickness $\mathrm{h}=2 \mathrm{~mm}$ ) provides the antenna gain $30 \ldots 30.7 \mathrm{dBi}$ and radiation efficiency $50 \ldots 60 \%$ within $24-24.4 \mathrm{GHz}$ frequency range. The parameters of the second example are listed in the Table 1.

Table 1. Parameters of LWAA for $36.5-37.3 \mathrm{GHz}$

\begin{tabular}{|l|l|}
\hline Parameter & Value \\
\hline Aperture size $\mathrm{L}_{\mathrm{x}} \times \mathrm{L}_{\mathrm{y}}$ & $94 \mathrm{~mm} \times 97 \mathrm{~mm}$ \\
\hline DWG: Rogers RT/Duroid 5880 & $\varepsilon_{\mathrm{r}}=2.2 ; \mathrm{tg} \delta=0.0009$ \\
\hline DWG thickness & $1.52 \mathrm{~mm}$ \\
\hline Strip width and spacing $\left(\mathrm{d}_{\mathrm{x}}\right)$ & $3.1 \mathrm{~mm} ; 6.3 \mathrm{~mm}$ \\
\hline Central strip width & $5.2 \mathrm{~mm}$ \\
\hline Number of strips & 15 \\
\hline Number of stubs & 32 \\
\hline Stub size and spacing $\left(\mathrm{d}_{\mathrm{y}}\right)$ & $1.6 \mathrm{~mm} \times 0.8 \mathrm{~mm} ; 5.8 \mathrm{~mm}$ \\
\hline Input slot size & $7.2 \mathrm{~mm} \times 3.4 \mathrm{~mm}$ \\
\hline Beamwidth $(\mathrm{E}-, \mathrm{H}-$ plane $)$ & $4.5 \mathrm{deg} . \times 4.5 \mathrm{deg}$. \\
\hline Maximum gain & $30.5 \mathrm{dBi}$ \\
\hline Radiation efficiency $(\mathrm{min} / \mathrm{max})$ & $50 \% / 65 \%$ \\
\hline VSWR (min/max) & $1.1 / 1.55$ \\
\hline Sidelobe level & $\leq-12 \mathrm{~dB}$ \\
\hline
\end{tabular}


It is clearly seen, that the second proposed LWAA with almost the same size of the radiation aperture, demonstrates practically the same gain and radiation efficiency values as the first LWAA; however, the operating frequency range of the first LWAA is as twice as wide than the frequency range of the second LWAA. Nevertheless, the second LWAA has an obvious advantage as more compact, easy to fabricate and low cost.

One more example of the second LWAA designed for 81-82 GHz frequency range has been simulated recently. Figure 7 illustrates data on simulated characteristics of directivity and gain of the antenna with the aperture size of $40 \mathrm{~mm} \times 40$ $\mathrm{mm} \times 0,52 \mathrm{~mm}$ (made of Rogers RT/Duroid 5880).

It seen that the radiation efficiency of the antenna is almost the same and reaches $58 \%$ at $81.5 \mathrm{GHz}$.

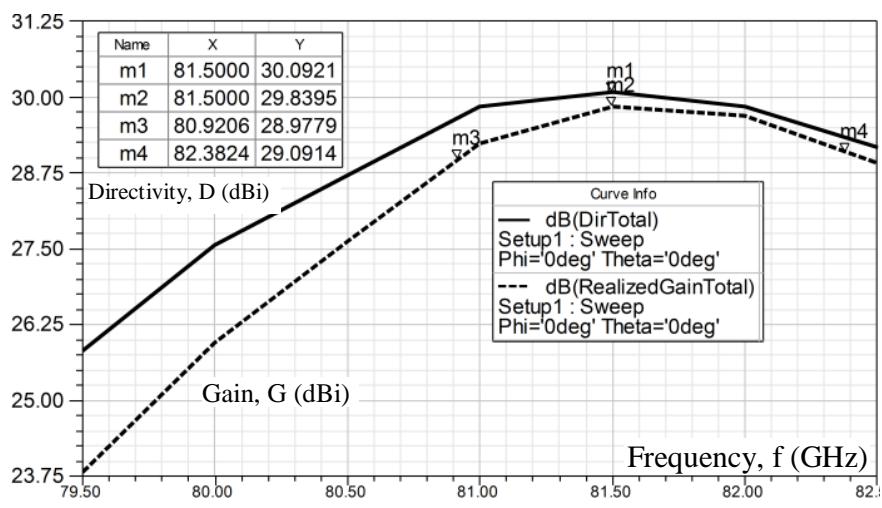

Figure 7. Simulated antenna directivity and gain

\section{CONCLUSION}

Two kinds of planar center-fed leaky-wave antenna arrays for linearly polarized broadside radiation are proposed and demonstrated.

Both kinds of antenna arrays contain a printed one-dimensional periodic metal strip grating, a grounded dielectric waveguide and a feeding device. The distinguished features of the antennas are low return loss and high gain at the frequency of the broadside radiation. The radiation efficiency of the antennas is above $50 \%$ within a wide range of the antenna gain, actually from 24 to $28-30 \mathrm{dBi}$ at frequencies up to $80-82 \mathrm{GHz}$.

\section{REFERENCES}

[1] Kao-Cheng Huang, Millimetre Wave Antennas for Gigabit Wireless Communications: a Practical Guide to Design and
Analysis in a System Context, JohnWiley \& Sons Ltd, 2008, pp. 48-94.

[2] M. Ando, "Planar Waveguide Arrays for Millimeter Wave Systems", IEICE Trans. Commun., Vol. E93-B, No.10, 2010, pp.2504-2513.

[3] A.A. Oliner, D.R. Jackson Ch.11: Leaky-Wave Antennas: Antenna Engineering Handbook, J.L.Volakis, Ed., New York, McGraw-Hill, 2007, pp. 254-358.

[4] T. Kawamura, A. Yamamoto, T. Teshirogi, Y. Kawahara, "Dual-Layer Parallel-Plate Waveguide Feed for Dielectric Leaky-Wave Antenna", ISAP2007, Aug. 21, 2007, No.1C4-5, pp. 47-65.

[5] T. Kawamura, T. Sakuma, A. Yamamoto, T. Teshirogi, "Experiments on Dielectric Leaky-Wave Antennas with Parallel-Plate Waveguide Feed", ISAP2008, 27-28 October 2008, No.1C06-1, pp. 36-42.

[6] P. Baccarelli, 1-D Periodic Leaky-Wave Antennas: Radiation Properties and Design Aspects. ESoA Course on Leaky Waves and Periodic Structures for Antenna Applications, "La Sapienza" University of Rome, Italy, 2011, April 26-29, pp. 324-329.

[7] V.P. Shestopalov, Physical Basics of Millimeter and Submillimeter Technique, Vol.1. Open Structures, Kiev, Naukova Dumka, 1985, pp. 257-291.

[8] Patent No.2435260 C2, H01Q13/00, Russian Federation. Planar Antenna / Nechaev J. B. (RU), Klimov A.I. (RU), Khokhlov N.S. (RU), Judin V.I. (RU), Rad'ko P.N. (RU). Publication Date 27.11.2011, Bulletin No.33.

[9] Patent Application No.2012144897, Russian Federation. Planar Leaky-Wave Antenna / Nechaev J. B. (RU), Klimov A.I. (RU), Borisov D.N. (RU), Judin V.I. (RU), Zolotukhin A.V. (RU). Priority Date 22.10.2012.

[10] Frank B. Gross, Frontiers in Antennas: Next Generation Design \& Engineering, NY, McGraw-Hill Comp., 2011, pp. 147-169.

[11]H.A. Kalhor, "Electromagnetic Scattering by a Dielectric Slab Loaded with a Periodic Array of Strips over a Ground Plane", IEEE Trans. Antennas and Propagat., Jan. 1988, N. 1, Vol. 36, pp. 147-151.

[12]J.A. Encinar, "Mode-Matching and Point-Matching Techniques Applied to the Analysis of Metal-Strip-Loaded Dielectric Antennas", IEEE Trans. Antennas and Propagat., Sep. 1990, N. 9, Vol. 38, pp. 1405-1412.

[13] Chang-Won Lee, Hyon Son., "Analysis of Electromagnetic Scattering by Periodic Strip Grating on a Grounded Dielectric/Magnetic Slab for Arbitrary Plane Wave Incidence Case", IEEE Trans. Antennas and Propagat., Sep. 1999, N. 9, Vol. 47, pp. 1386-1392.

[14]P. Baccarelli, P. Burghignoli, F. Frezza, A. Galli, P. Lampariello, G. Lovat, S. Paulotto, "Modal Properties of Surface and Leaky Waves Propagating at Arbitrary Angles Along a Metal Strip Grating on a Grounded Slab", IEEE Trans. Antennas and Propagat., Jan. 2005, N. 1, Vol. 53, pp. $36-46$.

[15] M. Matsumoto, M. Tsutsumi, N. Kumagai, "Radiation of Millimeter Waves from a Leaky Dielectric Waveguide with a Light-Induced Grating Layer", IEEE Trans. Microwave Theory and Techn.., Nov. 1987, N. 11, Vol. MTT-35, pp. 1033-1041. 